\section{Human Amniotic Membrane as a Matrix for Endothelial Differentiation of VEGF-Treated Dental Stem Cells}

By: Hashim, SNM (Hashim, Siti Nurnasihah Md) ${ }^{[1]}$; Yusof, MFH (Yusof, Muhammad Fuad Hilmi) ${ }^{[1]}$; Zahari, W (Zahari, Wafa') ${ }^{[1]} ;$ Chandra, H (Chandra, Hamshawagini) $^{[1]}$; Noordin, KBAA (Noordin, Khairul Bariah Ahmad Amin) ${ }^{[1]}$; Kannan, TP (Kannan, Thirumulu Ponnuraj) ${ }^{[1,2]}$; Hamid, SSA (Hamid, Suzina Sheikh Abdul) ${ }^{[3]}$; Mokhtar, KI (Mokhtar, Khairani Idah) ${ }^{[4]}$; Azlina, A (Azlina, Ahmad) ${ }^{[1,2]}$

View Web of Science ResearcherID and ORCID

CELLULAR AND MOLECULAR BIOENGINEERING

Volume: 12 Issue: 6 Pages: 599-613

DOI: $10.1007 /$ s12195-019-00596-x

Published: DEC 2019

Document Type: Article

View Journal Impact

\section{Abstract}

Introduction Endothelial cells cover the surface of the capillary wall and literature review has cemented its angiogenic roles in wound healing and tissue regeneration. However, the angiogenic in vitro models available are inadequate to understand the endothelial differentiation process. Methods $\mathrm{A}$ construct was made using human amniotic membrane (HAM) as a matrix to assist the dental stem cells to differentiate into endothelial-like cells. This study aimed to assess the biological interaction between stem cells from human exfoliated deciduous teeth (SHED) and the stromal side (SS) of the glycerol-preserved HAM in angiogenic-induced environment media using VEGF. The changes were evaluated through cell morphology, migration, as well as gene expression level. Results There were morphological changes observed in SHED in angiogenic-induced media. SHED appeared to be differentiated from fibroblast-like cells to a new structure, mimicking endothelial-like structure through microscopy analysis. Besides, the cross-section of the construct revealed that the cells seeded on the matrix were able to maintain its monolayer structure at day 1,7 and 14 but infiltrated into the HAM at day 21 , suggesting cell migration. The cells were also able to maintain its stemness (Nestin, Nanog and CD29) and at the same time express the angiogenic markers (IL-8, VEGF and MMP-2). Conclusion HAM promotes SHED proliferation, migration and has the potential as a differentiating matrix for endotheliallike cells.

\section{Keywords}

Author Keywords: Angiogenic; Endothelial differentiation; Extracellular matrix; Regenerative medicine; Stem cells from human exfoliated deciduous teeth; Vascular endothelial growth factor

KeyWords Plus: EXFOLIATED DECIDUOUS TEETH; MESENCHYMAL STROMAL CELLS; PULP; INTERLEUKIN-8; ANGIOGENESIS; SURVIVAL; ADHESION; BIOLOGY; GROWTH; NANOG

\section{Author Information}

Reprint Address: Azlina, A (reprint author)

+ Univ Sains Malaysia, Sch Dent Sci, Hlth Campus, Kubang Kerian 16150, Kelantan, Malaysia.

\section{Addresses:}

+ [1] Univ Sains Malaysia, Sch Dent Sci, HIth Campus, Kubang Kerian 16150, Kelantan, Malaysia

+ [2] Univ Sains Malaysia, Sch Med Sci, Human Genome Ctr, Hlth Campus, Kubang Kerian 16150, Kelantan, Malaysia

+ [ 3] Univ Sains Malaysia, Sch Med Sci, Tissue Bank, Hlth Campus, Kubang Kerian 16150, Kelantan, Malaysia

+ [4] Int Islamic Univ Malaysia, Kulliyyah Dent, Bandar Indera Mahkota Campus, Kuantan 25200, Pahang, Malaysia

E-mail Addresses: azlinakb@usm.my

Funding

\begin{tabular}{|c|c|c|}
\hline Funding Agency & Show details & Grant Number \\
\hline Universiti Sains & & 1001/PPSG/813075 \\
\hline
\end{tabular}

View funding text

Publisher

SPRINGER, 233 SPRING ST, NEWYORK, NY 10013 USA

\section{Journal Information}

Impact Factor: Journal Citation Reports

\section{Categories / Classification}

Research Areas: Cell Biology; Biophysics; Engineering

Web of Science Categories: Cell \& Tissue Engineering; Biophysics; Cell Biology; Engineering, Biomedical

\section{Citation Network}

In Web of Science Core Collection

1

Times Cited

Create Citation Alert

All Times Cited Counts

1 in All Databases

See more counts

\section{0}

Cited References

View Related Records

Most recently cited by:

Yusof, Muhammad Fuad Hilmi; Hashim, Siti Nurnasihah Md; Zahari, Wafa'; et al. Amniotic Membrane Enhance the Effect of Vascular Endothelial Growth Factor on the Angiogenic Marker Expression of Stem Cells from Human Exfoliated Deciduous Teeth.

APPLIED BIOCHEMISTRY AND BIOTECHNOLOGY (2020)

View All

\section{Use in Web of Science} Web of Science Usage Count

\section{8} 8

Last 180 Days Since 2013

Learn more

\section{This record is from}

Web of Science Core Collection

- Science Citation Index Expanded

Suggest a correction

If you would like to improve the quality of the data in this record, please suggest a correction. 


\section{Cited References: 50}

1. Term amniotic membrane is a high throughput source for multipotent mesenchymal stem cells with the ability to differentiate into endothelial cells in vitro Times Cited: 267 By: Alviano, Francesco; Fossati, Valentina; Marchionni, Cosetta; et al.

BMC DEVELOPMENTAL BIOLOGY Volume: 7 Article Number: 11 Published: FEB 212007

2. The role of ECM proteins and protein fragments in guiding cell behavior in regenerative medicine

Times Cited: 84

By: Barker, Thomas $\mathrm{H}$

BIOMATERIALS Volume: 32 Issue: 18 Pages: 4211-4214 Published: JUN 2011

3. Endothelial Differentiation of SHED Requires MEK1/ERK Signaling

Times Cited: 51

By: Bento, L. W.; Zhang, Z.; Imai, A.; et al.

JOURNAL OF DENTAL RESEARCH Volume: 92 Issue: 1 Pages: 51-57 Published: JAN 2013

4. Development of a Small Diameter Vascular Graft Using the Human Amniotic Membrane

Times Cited: 5

By: Brennan, Jaclyn A.; Arrizabalaga, Julien H.; Nollert, Matthias U.

CARDIOVASCULAR ENGINEERING AND TECHNOLOGY Volume: 5 Issue: 1 Pages: 96-109 Published: MAR 2014

5. Angiogenic Properties of Human Dental Pulp Stem Cells

Times Cited: 94

By: Bronckaers, Annelies; Hilkens, Petra; Fanton, Yanick; et al.

PLOS ONE Volume: 8 Issue: 8 Article Number: e71104 Published: AUG 72013

6. Mechanisms of angiogenesis and arteriogenesis

Times Cited: 2,888

By: Carmeliet, $P$

NATURE MEDICINE Volume: 6 Issue: 4 Pages: 389-395 Published:APR 2000

7. Fate of the mammalian cranial neural crest during tooth and mandibular morphogenesis

Times Cited: 899

By: Chai, Y; Jiang, XB; Ito, Y; et al.

DEVELOPMENT Volume: 127 Issue: 8 Pages: 1671-1679 Published: APR 2000

8. Human postnatal dental pulp cells co-differentiate into osteoblasts and endotheliocytes: a pivotal synergy leading to adult bone tissue formation

Times Cited: 314

By: d'Aquino, R.; Graziano, A.; Sampaolesi, M.; et al.

CELL DEATH AND DIFFERENTIATION Volume: 14 Issue: 6 Pages: 1162-1171 Published: JUN 2007

9. Minimal criteria for defining multipotent mesenchymal stromal cells. The International Society for Cellular Therapy position statement

Times Cited: 8,180

By: Dominici, M.; Le Blanc, K.; Mueller, l.; et al.

CYTOTHERAPY Volume: 8 Issue: 4 Pages: 315-317 Published: AUG 2006

10. Matrix metalloproteinase-2 regulates vascular patterning and growth affecting tumor cell survival and invasion in GBM

Times Cited: 64

By: Du, Rose; Petritsch, Claudia; Lu, Kan; et al.

NEURO-ONCOLOGY Volume: 10 Issue: 3 Pages: 254-264 Published: JUN 2008

11. Angiogenic Potential of Cryopreserved Amniotic Membrane Is Enhanced Through Retention of All Tissue Components in Their Native State

Times Cited: 25

By: Duan-Arnold, Yi; Uveges, Thomas E.; Gyurdieva, Alexandra; et al.

ADVANCES IN WOUND CARE Volume: 4 Issue: 9 Pages: 513-522 Published: SEP 12015

12. Amniotic membrane can be a valid source for wound healing

Times Cited: 9

By: ElHeneidy, Hossam; Omran, Eman; Halwagy, Ahmed; et al.

INTERNATIONAL JOURNAL OF WOMENS HEALTH Volume: 8 Pages: 225-231 Published: 2016

13. Development of a Cost-Effective and Simple Protocol for Decellularization and Preservation of Human Amniotic Membrane as a Soft Tissue Replacement and Delivery System for Bone Marrow Stromal Cells

By: Gholipourmalekabadi, Mazaher; Mozafari, Masoud; Salehi, Mohammad; et al.

ADVANCED HEALTHCARE MATERIALS Volume: 4 Issue: 6 Pages: 918-926 Published: APR 222015

14. Differences between human and mouse embryonic stem cells

Times Cited: 493

By: Ginis, I; Luo, YQ; Miura, T; et al.

DEVELOPMENTAL BIOLOGY Volume: 269 Issue: 2 Pages: 360-380 Published: MAY 132004

15. Stem cell properties of human dental pulp stem cells

Times Cited: 1,149

By: Gronthos, S; Brahim, J; Li, W; et al.

JOURNAL OF DENTAL RESEARCH Volume: 81 Issue: 8 Pages:531-535 Published:AUG 2002 
17. Optimization of Amniotic Membrane (AM) Denuding for Tissue Engineering

Times Cited: 70

By: Hopkinson, Andrew; Shanmuganathan, Vijay A.; Gray, Trevor; et al.

TISSUE ENGINEERING PART C-METHODS Volume: 14 Issue: 4 Pages: 371-381 Published: DEC 2008

18. Mesenchymal Stem Cells Derived from Dental Tissues vs. Those from Other Sources: Their Biology and Role in Regenerative Medicine

Times Cited: 902

By: Huang, G. T. -J.; Gronthos, S.; Shi, S

JOURNAL OF DENTAL RESEARCH Volume: 88 Issue: 9 Pages: 792-806 Published: SEP 2009

19. Human amniotic membrane as a delivery matrix for articular cartilage repair

By: Jin, Cheng Zhe; Park, So Ra; Choi, Byung Hyune; et al.

TISSUE ENGINEERING Volume: 13 Issue: 4 Pages: 693-702 Published:APR 2007

20. Isolation and in vitro characterisation of dental pulp stem cells from natal teeth

Times Cited: 111

By: Karaoz, Erdal; Dogan, Burcu Nur; Aksoy, Ayca; et al.

HISTOCHEMISTRY AND CELL BIOLOGY Volume: 133 Issue: 1 Pages: 95-112 Published: JAN 2010

21. Isolation and characterization of a population of immature dental pulp stem cells expressing OCT-4 and other embryonic stem cell markers

By: Kerkis, Irina; Kerkis, Alexandre; Dozortsev, Dmitri; et al.

CELLS TISSUES ORGANS Volume: 184 Issue: 3-4 Pages: 105-116 Published: 2006

22. Stem Cells in Dental Pulp of Deciduous Teeth

Times Cited: 77

By: Kerkis, Irina; Caplan, Arnold I.

TISSUE ENGINEERING PART B-REVIEWS Volume: 18 Issue: 2 Pages: 129-138 Published: APR 2012

23. In Vivo Angiogenic Capacity of Stem Cells from Human Exfoliated Deciduous Teeth with Human Umbilical Vein Endothelial Cells

Times Cited: 9

By: Kim, Ji-Hye; Kim, Gee-Hye; Kim, Jae-Won; et al.

MOLECULES AND CELLS Volume: 39 Issue: 11 Pages: 790-796 Published: NOV 302016

24. INTERLEUKIN-8 AS A MACROPHAGE-DERIVED MEDIATOR OF ANGIOGENESIS

Times Cited: 1,744

By: KOCH, AE; POLVERINI, PJ; KUNKEL, SL; et al.

SCIENCE Volume: 258 Issue: 5089 Pages: 1798-1801 Published: DEC 111992

25. NANOG induction of fetal liver kinase-1 (FLK1) transcription regulates end othelial cell proliferation and angiogenesis

By: Kohler, Erin E.; Cowan, Colleen E.; Chatterjee, Ishita; et al.

BLOOD Volume: 117 Issue: 5 Pages: 1761-1769 Published: FEB 32011

26. Interleukin-1 beta Induces Angiogenesis and Innervation in Human Intervertebral Disc Degeneration

Times Cited: 104

By: Lee, Jae Man; Song, Ji Ye; Baek, MinJung; et al.

JOURNAL OF ORTHOPAEDIC RESEARCH Volume: 29 Issue: 2 Pages: 265-269 Published: FEB 2011

27. IL-8 directly enhanced endothelial cell survival, proliferation, and matrix metalloproteinases production and regulated angiogenesis

Times Cited: 787

By: Li, AH; Dubey, S; Varney, ML; et al.

JOURNAL OF IMMUNOLOGY Volume: 170 Issue: 6 Pages: 3369-3376 Published: MAR 152003

28. Nestin-mediated cytoskeletal remodeling in endothelial cells: novel mechanistic insight into VEGF-induced cell migration in angiogenesis

Times Cited: 18

By: Liang, Zhen-wei; Wang, Zheng; Chen, Hui; et al.

AMERICAN JOURNAL OF PHYSIOLOGY-CELL PHYSIOLOGY Volume: 308 Issue: 5 Pages: C349-C358 Published: MAR 12015

29. Amniotic membrane use in dermatology

Times Cited: 37

By: Lo, Venetia; Pope, Elena

INTERNATIONAL JOURNAL OF DERMATOLOGY Volume: 48 Issue: 9 Pages: 935-940 Published: SEP 2009

30. Filopodia: molecular architecture and cellular functions

Times Cited: 897 By: Mattila, Pieta K.; Lappalainen, Pekka

NATURE REVIEWS MOLECULAR CELL BIOLOGY Volume: 9 Issue: 6 Pages: 446-454 Published: JUN 2008

\section{Showing $\mathbf{3 0}$ of $\mathbf{5 0} \quad$ View All in Cited References page}

